\title{
Ordonnancement des mesures pour la détection et la localisation de défauts dans un système contrôlé en réseau
}

\author{
Mohamed Amine Sid ${ }^{1,2}$, Samir Aberkane ${ }^{1,2}$, Dominique Sauter ${ }^{1,2}$, Didier Maquin ${ }^{1,2}$ \\ ${ }^{1}$ Centre de Recherche en Automatique de Nancy, \\ UMR 7039 - Université de Lorraine, CNRS \\ 2, Avenue de la forêt de Haye. 54516 Vandœuvre, France. \\ prenom.nom@univ-lorraine.fr \\ ${ }^{2}$ CNRS, CRAN, UMR 7039, France.
}

\begin{abstract}
Résumé- Ce travail porte sur le problème de détection et d'isolation de défauts multiples sous contraintes de communication. En particulier, nous considérons le problème de la co-conception de l'ordonnancement des tâches de transmission des mesures en provenance des capteurs et de la détection et la localisation des défauts dans le cas de bande passante limitée du média de transmission. Le filtre isolateur proposé est une forme particulière du filtre de Kalman. L'ordonnancement des mesures et le filtre sont calculés pour assurer l'isolabilité des défauts et minimiser la trace de la matrice de covariance de l'erreur d'estimation.
\end{abstract}

Mots-clés- Ordonnancement de mesures, détection et isolation de défauts, co-conception, systèmes contrôlés en réseaux.

\section{INTRODUCTION}

L'étude des systèmes contrôlés en réseau (SCR) est devenue un axe de recherche très actif au cours de la dernière décennie. Les avantages de cette architecture sont liés au partage du réseau à travers lequel les capteurs, les actionneurs et les calculateurs communiquent. Par rapport aux systèmes câblés traditionnels, l'utilisation d'un réseau de communication réduit les coûts de câblage, facilite la supervision et permet d'augmenter la versatilité du système contrôlé. Cependant, de nouvelles contraintes surviennent lorsque les informations des capteurs et des contrôleurs sont transmises à travers le réseau. Ces contraintes sont classées selon cinq catégories [5], [20] :

1. Erreurs de quantification des signaux transmis à travers le réseau due à la longueur finie du mot (taille des paquets);

2. Perte de paquets dans les réseaux non-fiables;

3. Intervalles d'échantillonnage/transmission variables;

4. Retard de communication variable;

5. Contraintes d'accès au média due à la bande passante limitée liée au partage du réseau par plusieurs nœuds et le fait qu'un seul nœud à la fois est autorisé à transmettre son paquet.

6. Consommation d'énergie élevée notamment pour les systèmes contrôlés en réseau sans fil.

La présence de ces contraintes engendre la dégradation des performances du système de diagnostic et nécessite des algorithmes robustes vis-à-vis des contraintes de communication. Toutes ces contraintes peuvent exister dans un réseau de communication, mais seules certaines d'entre elles sont prises en compte dans les travaux dédiés aux SCR, en particulier l'effet du retard induit, la perte de paquets et l'effet de l'échantillonnage. Le problème du retard est considéré par exemple dans [18], [11], [19], [5]. Dans [12], les auteurs proposent la conception d'un système robuste de détection de défaut lorsque les retards de transmission sont important, mais dans ce cas, le découplage total de l'effet de défaut de l'entrée inconnue est impossible. Un filtre de Kalman adaptatif est proposé dans [1] pour la minimisation de l'effet du retard induit sur le résidu. La problématique de détection de défaut pour un SCR avec des entrées et des mesures retardées est traitée dans [8]. Dans [19], [21], le système de détection de défaut pour un SCR avec pertes de paquet est conçu après la modélisation du SCR par un système linéaire à saut markovien. Le problème de détection de défaut sous contraintes de communication multiples est considéré dans [11], [16], [4]. Notons, qu'à la connaissance des auteurs, le problème de localisation des défauts dans les SCR n'a pas été étudié auparavant.

L'objectif de cet article est de traiter le problème de détection et de localisation de défauts multiples sous contraintes de communication. Plus précisément, nous allons considérer la contrainte d'accès au média. Dans ce cas, le réseau partagé ne peut supporter qu'un nombre limité de communications entre les éléments du système. Il est alors nécessaire de concevoir un module de détection et de localisation de défaut en conjonction avec une une politique de communication définissant les instants auxquels les capteurs sont autorisés à accéder au média. La séquence de communication précise les capteurs qui sont capables d'envoyer leurs mesures vers le filtre de détection à chaque instant d'échantillonnage. Cela conduit naturellement à considérer une problématique de co-conception (codesign), c'est-à-dire, la conception simultanée d'un filtre de détection-localisation et de la séquence d'ordonnancement des mesures. La séquence d'ordonnancement des mesures et le filtre proposés sont ici conçus de sorte à assurer l'isolabilité du défaut et la minimisation de l'effet de bruit. La suite de cet article est organisée comme suit : la section II donne une formulation complète du problème. Dans la section III, les résultats essentiels sont présentés. Le problème d'ordonnancement des mesures est présenté dans la section IV. Un exemple numérique est donné dans la section $\mathrm{V}$ pour illus- 


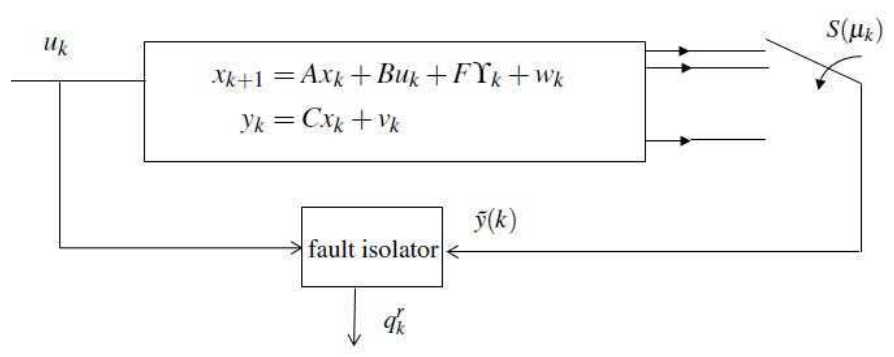

Fig. 1. Système de diagnostic

trer l'efficacité de la méthode de co-conception proposée. Finalement, des conclusions et quelques directions de recherche sont énoncées dans la section VI.

\section{Formulation DU PROBLÈme}

Considérons le système illustré à la figure 1. La représentation d'état du système sujette à un défaut d'actionneur et/ou défaut de composants s'écrit :

$$
\begin{cases}x_{k+1} & =A x_{k}+B u_{k}+F \Upsilon_{k}+w_{k} \\ y_{k} & =C x_{k}+v_{k}\end{cases}
$$

où $x_{k} \in \mathbb{R}^{n}$ est le vecteur d'état, $u_{k}$ est la commande, $F=\left[f_{1}, f_{2}, \ldots, f_{q}\right] \in \mathbb{R}^{n \times q}$ est la matrice de distribution des défauts, $\Upsilon_{k}=\left[\Upsilon_{k}^{1}, \Upsilon_{k}^{2}, \ldots, \Upsilon_{k}^{q}\right]^{T} \in \mathbb{R}^{q}$ est le vecteur de défaut $y_{k} \in \mathbb{R}^{m}$ est le vecteur de mesures. L'état initial $x_{0}$, les perturbations $w_{k}$ et le bruit de mesure $v_{k}$ sont des processus aléatoires blancs et Gaussiens non corrélés avec une moyenne nulle, $x_{0} \sim \mathcal{N}\left(\bar{x}_{0}, \bar{P}_{0}\right), w_{k} \sim \mathcal{N}(0, W)$ et $v_{k} \sim \mathcal{N}\left(0, I_{m}\right)$ respectivement, où $\bar{P}_{0}, W$ sont des matrices définies positives.

L'objectif principal de cet article est la conception d'un filtre de détection et d'isolation de défaut prenant en compte les contraintes de communication induites par le réseau de communication partagé. Plus précisément, la contrainte de communication que nous traitons dans cet article est mentionné dans la littérature comme la contrainte d'accès au média. Dans ce cas, le réseau partagé ne peut supporter qu'un nombre limité de communications simultanées entre les éléments du système.

\section{A. Séquence de communication}

Nous allons considérer que le réseau de communication reliant les capteurs et le filtre de détection qui engendre un résidu possède $b$ canaux de sortie, avec :

$$
1 \leq b \leq m
$$

A chaque instant, $b$ capteurs parmi $m$ peuvent accéder à ces canaux pour communiquer avec le générateur de résidus tandis que les autres restent en attente. On a $\sigma=C_{m}^{b}=\frac{m !}{b !(m-b) !}$ configurations possibles. On introduit l'application : $\mu_{k}: Z \rightarrow \mathcal{M}=\{1, \ldots, \sigma\}$, qui détermine, à chaque instant d'échantillonnage, l'indice du groupe de capteurs correspondant. Nous appelons cette application une configuration de commutation de capteurs.
A la figure 1 , le signal $\tilde{y}_{k} \in \mathbb{R}^{b}$ est lié à $y_{k}$ par la relation suivante : $\tilde{y}_{k}=S\left(\mu_{k}\right) y_{k}$. La matrice de commutation $S\left(\mu_{k}\right) \in \mathbb{R}^{b \times m}$ est utilisée pour sélectionner le sousensemble de mesures qui seront transmises au contrôleur à chaque instant d'échantillonnage $k$. Chaque ligne possède donc un seul élément qui vaut 1 , les autres termes sont des 0 . Ce sous-ensemble est indexé par les valeurs de la configuration de commutation $\mu_{k}$. Considérant la bande passante limitée du média de communication, le modèle étendu du système est décrit par :

$$
\left\{\begin{array}{l}
x_{k+1}=A x_{k}+B u_{k}+F \Upsilon_{k}+w_{k} \\
\tilde{y}_{k}=\mathcal{C}_{\mu_{k}} x_{k}+\tilde{v}_{k}
\end{array}\right.
$$

où $\mathcal{C}_{\mu_{k}}=S\left(\mu_{k}\right) C$ and $\tilde{v}_{k}=S\left(\mu_{k}\right) v_{k}$.

\section{B. Filtre de détection}

Le filtre de détection et de localisation proposé dans cet article est une version modifiée du filtre proposé dans [7]. Sa représentation d'état est donnée par :

$$
\begin{cases}\hat{x}_{k+1} & =A \hat{x}_{k}+B u_{k}+K_{k}\left(\tilde{y}_{k}-\hat{y}_{k}\right) \\ \hat{y}_{k} & =\mathcal{C}_{\mu_{k}} \hat{x}_{k}\end{cases}
$$

où $\hat{x}_{k}$ et $\hat{y}_{k}$ désignent les vecteurs d'état et de sortie estimés, respectivement. Ce filtre estimateur permet d'engendrer un résidu qui sera analysé pour détecter et localiser les défauts.

Il est important de noter que dans le contexte considéré dans cet article, le gain du filtre $K_{k}$ et la configuration de commutation $\mu_{k}$ sont les deux paramètres de conception.

\section{RÉSUlTATS PRINCIPAUX}

Dans cette section, nous introduisons nos résultats principaux. Nous allons d'abord rappeler quelques définitions de base qui seront utilisées par la suite.

Définition 1. [7] Le système linéaire stochastique (1) possède les indices de détectabilité de défaut $\rho=\left\{\rho_{1}, \rho_{2}, \ldots, \rho_{q}\right\}$ si $\rho_{i}=\min \left\{\nu: C A^{\nu-1} f_{i} \neq 0, \nu=\right.$ $1,2, \ldots, q\}$.

Définition 2. La matrice de détectabilité à temps variant associée au système étendu est définie comme suit

$$
D_{\mu_{k}}=\mathcal{C}_{\mu_{k}} \Psi
$$

où

$$
\Psi=\left[A^{\rho_{1}-1} f_{1}, A^{\rho_{2}-1} f_{2}, \ldots, A^{\rho_{s}-1} f_{s}\right]
$$

Soit $s=\max \left\{\rho_{i}, \quad i=1,2, \ldots, q\right\}$ la valeur maximale des indices de détectabilité de défauts. On définit $\bar{\Upsilon}_{k}=\left[\bar{\Upsilon}_{k}^{1 T}, \bar{\Upsilon}_{k}^{2 T}, \ldots, \bar{\Upsilon}_{k}^{s T}\right]^{T}, \bar{F}=\left[\bar{F}_{1}, \bar{F}_{2}, \ldots, \bar{F}_{s}\right]$, où $\bar{\Upsilon}_{i} \in \mathbb{R}_{i}^{q}$ représente le sous-ensemble des défauts ayant un indice de détectabilité $\rho_{i}$ et $\bar{F}_{i} \in \mathbb{R}^{n \times q_{i}}$ leur matrice de distribution. Le système étendu peut être réécrit sous la forme équivalente :

$$
\left\{\begin{array}{l}
x_{k+1}=A x_{k}+B u_{k}+\bar{F} \bar{\Upsilon}_{k}+w_{k} \\
\tilde{y}_{k}=\mathcal{C}_{\mu_{k}} x_{k}+\tilde{v}_{k}
\end{array}\right.
$$


Considérons le filtre donné par (4). La dynamique de l'erreur d'estimation $e_{k}=x_{k}-\hat{x}_{k}$ et le résidu de sortie $q_{k}=\tilde{y}_{k}-\hat{y}_{k}$ sont donnés par :

$$
\begin{cases}e_{k+1} & =\left(A-K_{k} \mathcal{C}_{\mu_{k}}\right) e_{k}+\bar{F} \bar{\Upsilon}_{k}-K_{k} \tilde{v}_{k}+w_{k} \\ q_{k} & =\mathcal{C}_{\mu_{k}} e_{k}+\tilde{v}_{k}\end{cases}
$$

D'après le principe de superposition, l'effet d'un défaut additif survenant à l'instant $r$ (avec $k>r+s)$, sur le résidu de sortie $q_{k}$ est donné par :

$$
q_{k}=\bar{q}_{k}+\bar{\rho}_{k, r}\left[\begin{array}{llllll}
\bar{\Upsilon}_{r}^{T} & \ldots & \bar{\Upsilon}_{k-s}^{T} & \cdots & \bar{\Upsilon}_{k-2}^{T} & \bar{\Upsilon}_{k-1}^{T}
\end{array}\right]^{T}
$$

avec

$$
\begin{aligned}
& \bar{\rho}_{k, r}=\mathcal{C}_{\mu_{k}}\left[\begin{array}{llllll}
G_{k-1, r} \bar{F} & \cdots & G_{k-1, k-(s-1)} \bar{F} & \cdots & G_{k-1} \bar{F} & \bar{F}
\end{array}\right] \\
& G_{k-1, k-j}=G_{k-1} G_{k-2} \cdots G_{k-j} \\
& G_{k}=\left(\begin{array}{lll}
\left.A-K_{k} \mathcal{C}_{\mu_{k}}\right)
\end{array}\right.
\end{aligned}
$$

où $\bar{q}_{k}$ correspond au résidu de sortie pour le cas non défaillant.

En suivant les arguments développés dans [7], on peut énoncer les résultats suivants :

\section{Proposition 1. (Condition d'isolabilité de défaut)} Sous la condition $\operatorname{rang}\left(\mathcal{D}_{\mu_{k}}\right)=q$, la solution de la contrainte algébrique : $\left(A-K_{k} \mathcal{C}_{\mu_{k}}\right) \bar{\Psi}=0$ peut être paramétrée par $K_{k}=\omega \Pi_{\mu_{k}}+\bar{K}_{k} \Sigma_{\mu_{k}}$ avec

$$
\Sigma_{\mu_{k}}=\alpha_{\mu_{k}}\left(I_{m}-D_{\mu_{k}} \Pi_{\mu_{k}}\right), \Pi_{\mu_{k}}=D_{\mu_{k}}^{+} \text {et } \omega=A \bar{\Psi}
$$

avec $\bar{\Psi}=\left[\bar{F}_{1}, A \bar{F}_{2}, \ldots, A^{s-1} \bar{F}_{s}\right] . \bar{K}_{k} \in \mathbb{R}^{n \times(b-q)}$ est le gain réduit à déterminer, $D_{\mu_{k}}^{+}$est la pseudo-inverse de MoorePenrose de la matrice $D_{\mu_{k}}$ et $\alpha_{\mu_{k}}$ est une matrice arbitraire déterminée de façon à ce que la matrice $\Sigma_{\mu_{k}}$ soit de plein rang ligne.

Sous ces conditions, le résidu $q_{k}$ est donné par

$$
q_{k}=\bar{q}_{k}+D_{\mu_{k}}\left[\begin{array}{llll}
\bar{\Upsilon}_{k-1}^{1 T} & \bar{\Upsilon}_{k-2}^{2 T} & \cdots & \bar{\Upsilon}_{k-s}^{s T}
\end{array}\right]^{T}
$$

Remarque 1. Dans le résultat donné ci-dessus, il est important de noter que la matrice $\mathcal{C}_{\mu_{k}}$ dépend de la configuration de commutation $\mu_{k}$. Les séquences de commutation qui ne vérifient pas la condition de rang de la Proposition 1 doivent être exclues. Par conséquent, définissons l'ensemble des configurations de commutation admissibles $\Xi^{*}$ donné par

$$
\Xi^{*}=\left\{\mu_{k}: Z \rightarrow \mathcal{M}^{*} \subseteq \mathcal{M}\right\}
$$

où $\mathcal{M}^{*}$ contient les indices correspondant à des configurations de capteurs (produites par des matrices correspondantes $\left.\mathcal{C}_{\mu_{k}}\right)$ qui vérifient la condition $\operatorname{rang}\left(\mathcal{D}_{\mu_{k}}\right)=q$. Le filtre isolateur de défaut est conçu en calculant le paramètre libre $\bar{K}_{k}$ de sorte que la trace de la matrice de covariance $\bar{P}_{k}=E\left(\bar{e}_{k} \bar{e}_{k}^{T}\right)$ soit minimale.

Proposition 2. (Conception du filtre isolateur de défaut) Pour une configuration de commutation donnée $\mu_{k} \in \Xi^{*}$, Le filtre de détection de défaut proposé est décrit par les relations suivantes :

$$
\hat{x}_{k+1}=A \hat{x}_{k}+B u_{k}+\omega q_{k}^{r}+\bar{K}_{\mu_{k}} \gamma_{k}
$$

$$
\begin{aligned}
\bar{P}_{k+1}= & \left(\bar{A}_{\mu_{k}}-\bar{K}_{\mu_{k}} \bar{C}_{\mu_{k}}\right) \bar{P}_{k}\left(\bar{A}_{\mu_{k}}-\bar{K}_{\mu_{k}} \bar{C}_{\mu_{k}}\right)^{T} \\
& \quad+\bar{K}_{\mu_{k}} \bar{V}_{\mu_{k}} \bar{K}_{\mu_{k}}^{T}+\bar{W}_{\mu_{k}} \\
= & \phi_{\mu_{k}}\left(\bar{P}_{k}\right) \\
\bar{K}_{\mu_{k}}= & \bar{A}_{\mu_{k}}+\bar{P}_{k} \bar{C}_{\mu_{k}}^{T}\left(\bar{C}_{\mu_{k}} \bar{P}_{k} \bar{C}_{\mu_{k}}^{T}+\bar{V}_{\mu_{k}}\right)^{-1}
\end{aligned}
$$

avec

$$
\begin{aligned}
& \bar{A}_{\mu_{k}}=A-\omega \Pi_{\mu_{k}} \mathcal{C}_{\mu_{k}} \\
& \bar{C}_{\mu_{k}}=\Sigma_{\mu_{k}} \mathcal{C}_{\mu_{k}} \\
& \bar{V}_{\mu_{k}}=\Sigma_{\mu_{k}} \Sigma_{\mu_{k}}^{T} \\
& \bar{W}_{\mu_{k}}=W+\omega \Pi_{\mu_{k}} \Pi_{\mu_{k}}^{T} \omega^{T}
\end{aligned}
$$

et

$$
\begin{array}{r}
\gamma_{k}=\Sigma_{\mu_{k}}\left(\tilde{y}_{k}-\mathcal{C}_{\mu_{k}} \hat{x}_{k}\right) \\
q_{k}^{r}=\Pi_{\mu_{k}}\left(\tilde{y}_{k}-\mathcal{C}_{\mu_{k}} \hat{x}_{k}\right)
\end{array}
$$

Ce filtre possède les propriétés suivantes :

- $\gamma_{k}$ est découplé des défauts

- $q_{k}^{r}$ vérifie la relation

$$
q_{k}^{r}=\Pi_{\mu_{k}} \bar{q}_{k}+\left[\begin{array}{llll}
\bar{\Upsilon}_{k-1}^{1 T} & \bar{\Upsilon}_{k-2}^{2 T} & \cdots & \bar{\Upsilon}_{k-s}^{s T}
\end{array}\right]^{T}
$$

Chaque composante du résidu de sortie réduit $q_{k}^{r} \in \mathbb{R}^{q}$ n'étant sensible qu'à un seul défaut, l'analyse du vecteur résidu permet la localisation des défauts.

Preuve. La démonstration de cette proposition est similaire à la preuve du Théorème 3.1 dans [7].

L'évolution de la matrice de covariance donnée par l'équation de Riccati (13) dépend de la matrice de covariance initiale $P_{0}$ et de la configuration de commutation $\mu_{k}$. Par conséquent, en plus de la condition d'isolabilité (voir Proposition 1), la stratégie d'ordonnancement peut être générée pour optimiser l'évolution de la matrice de covariance. Ce point sera davantage exposé dans la section suivante

\section{ORDONNANCEMENT OPTIMAL À HORIZON FINI}

Le problème abordé ici est de choisir les $b$ capteurs qui doivent transmettre leurs mesures à chaque instant pour minimiser une fonction de la covariance de l'erreur d'estimation d'état. Définir la stratégie d'ordonnancement $s_{N}$ sur un horizon de $N$ échantillons équivaut à définir les valeurs de $\mu_{k}, k=0, \ldots, N-1$, ou de façon équivalente $s_{N}=\left[\begin{array}{llll}\mu_{0} & \mu_{1} & \ldots & \mu_{N-1}\end{array}\right]$. Soit $\mathcal{S}_{N}=\mathcal{M}^{N}$ l'ensemble de toutes les stratégies d'ordonnancement sur un horizon de taille $N$ et $\mathcal{S}_{N}^{*}$ l'ensemble des ordonnancements admissibles sur cet horizon (voir Remarque 1). Le problème d'ordonnancement optimal est formulé comme

$$
\min _{s_{N} \in \mathcal{S}_{N}^{*}} \mathcal{J}\left(s_{N}\right)
$$

où $\mathcal{J}\left(s_{N}\right)=\sum_{i=1}^{N} \operatorname{tr}\left(\bar{P}_{i}\right)=\sum_{i=0}^{N-1} \operatorname{tr}\left(\phi_{\mu_{i}}\left(\bar{P}_{i}\right)\right)$ et $\mu_{i}=s_{N}(i)$.

La recherche de cet optimum peut être effectuée de façon triviale en examinant tous les cas possibles. Cette 
énumération n'est envisageable que pour des horizons de faible taille à cause de l'explosion combinatoire du nombre de cas.

Pour analyser ce problème, les auteurs de [17] proposent de construire un arbre de recherche au sein duquel chaque

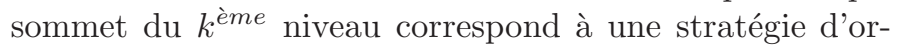
donnancement $s_{k}$ sur un horizon de $k$ échantillons. A chaque sommet est associé un couple de valeur $\left(\bar{P}_{k}^{s_{k}}, \gamma_{k}^{s_{k}}\right)$ où $\bar{P}_{k}^{s_{k}}$ est la matrice de variance obtenue en appliquant la stratégie d'ordonnancement $s_{k}$ et $\gamma_{k}^{s_{k}}=\mathcal{J}\left(s_{k}\right)$, le coût associé. Ces couples de valeurs peuvent aisément être calculés itérativement à l'aide de (14).

Une technique d'élagage de l'arbre de recherche peut ensuite être proposée, celle-ci réduisant clairement la complexité de la recherche. Rappelons tout d'abord deux définitions et un théorème introduits dans [17].

Définition 3. (Ensemble caractéristique) Soit la suite d'ensembles $\left\{\mathcal{H}_{k}\right\}_{k=0}^{N}$ définie récursivement par :

$$
\mathcal{H}_{k+1}=\pi_{\mathcal{M}^{*}}\left(\mathcal{H}_{k}\right) \text { avec } \mathcal{H}_{0}=\left\{\left(\bar{P}_{0}, \operatorname{tr}\left(\bar{P}_{0}\right)\right)\right\}
$$

avec

$\pi_{\mathcal{M}^{*}}(\mathcal{H})=\left\{\left(\phi_{i}(\bar{P}), \gamma+\operatorname{tr}\left(\phi_{i}(\bar{P})\right): \forall i \in \mathcal{M}^{*}, \forall(\bar{P}, \gamma) \in \mathcal{H}\right\}\right.$

Les ensembles $\mathcal{H}_{k}, k=1, \ldots N$, expriment la covariance de l'erreur d'estimation et la valeur de la fonction coût pour tous les ordonnancements possibles. Notez que la définition ci-dessus se distingue de l'originale dans [17] par l'utilisation de $\mathcal{M}^{*}$ au lieu de $\mathcal{M}$, permettant ainsi de satisfaire les contraintes d'isolabilité des défauts.

Définition 4. (Redondance algébrique) Un couple $(\bar{P}, \gamma) \in \mathcal{H}$, où $\mathcal{H}$ est un ensemble caractéristique (voir définition 3), est dit algébriquement redondant par rapport à $\mathcal{H} \backslash\{(\bar{P}, \gamma)\}$, s'il existe des constantes non négatives $\left\{\alpha_{i}\right\}_{i=1}^{l-1}$ telles que

$$
\sum_{i=1}^{l-1} \alpha_{i}=1 \text { et }\left[\begin{array}{cc}
\bar{P} & 0 \\
0 & \gamma
\end{array}\right] \geqslant \sum_{i=1}^{l-1} \alpha_{i}\left[\begin{array}{cc}
\bar{P}(i) & 0 \\
0 & \gamma(i)
\end{array}\right]
$$

où $l=\operatorname{card}(\mathcal{H})$ et $\{(\bar{P}(i), \gamma(i))\}_{i=1}^{l-1}$ est l'ensemble des éléments de $\mathcal{H} \backslash\{(\overline{\mathcal{P}}, \gamma)\}$.

Le théorème suivant fournit une condition qui caractérise les branches qui peuvent être élaguées sans pour autant éliminer la solution optimale du problème d'ordonnancement des mesures.

Theorème 1. Si le couple $(\bar{P}, \gamma) \in \mathcal{H}_{k}$ associé à un sommet particulier de l'arbre de recherche est algébriquement redondant, alors les branches issues de ce sommet (le sommet et tous ses descendants) peuvent être élagués sans pour autant éliminer la solution optimale de l'arbre de recherche.

Par manque de place, il n'est pas possible de décrire précisément l'algorithme d'élagage de l'arbre de recherche s'appuyant sur cette notion de couple algébriquement redondant. Le lecteur intéressé se réfèrera à l'article de Vitus et al. [17] pour la description de cet l'algorithme, référencé Algorithme 1.

Nous sommes maintenant en mesure de décrire l'algorithme d'ordonnancement des mesures sur un horizon fini assurant l'isolabilité des défauts et minimisant la variance du résidu.

\section{Algorithme 2. Ordonnancement des mesures sur un horizon fini}

1. $\mathcal{H}_{0}=\left\{\left(\bar{P}_{0}, \operatorname{tr}\left(\bar{P}_{0}\right)\right)\right\}$

2. for $k=1, \ldots, N$, do

$$
\mathcal{H}_{k}=\pi_{\mathcal{M}^{*}}\left(\mathcal{H}_{k-1}\right)
$$

Appliquer l'Algorithme 1 de [17] avec $\mathcal{H}_{k}$ end for

3. $\lambda^{*}=\underset{j \in\left\{1, \ldots, \operatorname{card}\left(\mathcal{H}_{N}\right)\right\}}{\arg \min } \mathcal{J}\left(\lambda\left(\mathcal{H}_{N}(j)\right)\right)$

Remarque 2. Dans [17], les auteurs ont proposé une solution sous-optimale s'appuyant sur le concept de $\epsilon$-redondance. La redondance algébrique énoncée dans la définition 4 est "relâchée" en remplaçant l'inégalité par

$$
\left[\begin{array}{cc}
\bar{P}+\epsilon I & 0 \\
0 & \gamma+\epsilon
\end{array}\right] \geqslant \sum_{i=1}^{l-1} \alpha_{i}\left[\begin{array}{cc}
\bar{P}(i) & 0 \\
0 & \gamma(i)
\end{array}\right]
$$

autorisant ainsi la prise en compte de la proximité numérique des contraintes. La prise en compte de cette $\epsilon$-redondance permet de diminuer de façon très importante la complexité de la résolution en élaguant de nombreuses branches supplémentaires.

\section{Ordonnancement Stochastique Des Mesures}

L'ordonnancement des mesures peut également être déterminé de façon stochastique comme le décrit la référence [7]. Dans ce cas, le choix des mesures transmises est aléatoire, la configuration de commutation $\mu_{k}^{*} \in \Xi^{*}$ est donc aléatoire, et l'on cherche à assurer des propriétés à la distribution de probabilité correspondante. Ici, on cherche à minimiser la covariance de l'erreur d'estimation en régime permanent. Comme cette variance $\bar{P}_{k}$ dépend de la configuration de commutation $\mu_{k}^{*}$ qui est aléatoire, $\bar{P}_{k}$ est également aléatoire. L'étude portera donc sur son espérance $E\left(\bar{P}_{k}\right)$ que l'on évaluera lorsque $k \rightarrow \infty$.

Rappelons que l'équation récurrente de Riccati pour un ordonnancement stochastique est donnée par

$$
\begin{aligned}
\bar{P}_{k+1}= & \left(\bar{A}_{\mu_{k}^{*}}-\bar{K}_{\mu_{k}^{*}} \bar{C}_{\mu_{k}^{*}}\right) \bar{P}_{k}\left(\bar{A}_{\mu_{k}^{*}}-\bar{K}_{\mu_{k}^{*}} \bar{C}_{\mu_{k}^{*}}\right)^{T} \\
& +\bar{K}_{\mu_{k}^{*}} \bar{V}_{\mu_{k}^{*}} \bar{K}_{\mu_{k}^{*}}^{T}+\bar{W}_{\mu_{k}^{*}} \\
= & \phi_{\mu_{k}^{*}}\left(\bar{P}_{k}\right)
\end{aligned}
$$

Supposons maintenant que, à chaque instant $k$, la configuration de commutation $\mu_{k}^{*}$ corresponde à la sélection d'un groupe de capteur indicé par $i, i \in\{1, \ldots, \sigma\}$ et que la probabilité de sélection de cette configuration s'exprime par :

$$
\mathcal{P}\left(\mu_{k}^{*}=i\right)=\pi_{k}^{i} \quad \text { pour } \quad i=1, \ldots, \sigma
$$


Supposons en outre que les probabilités $\pi_{k}^{i}$ tendent vers des valeurs constantes $\pi_{i}$ lorsque $k \rightarrow \infty$. Ainsi, nous intéressons à l'équation dynamique de $E\left(\bar{P}_{k}\right)$

$$
E\left[\bar{P}_{k+1}\right]=E\left[\phi_{\mu_{k}^{*}}\left(\bar{P}_{k}\right)\right]=\sum_{i=1}^{\sigma} \pi_{i} E\left[\phi_{i}\left(\bar{P}_{k}\right)\right]
$$

En général, il est impossible d'évaluer analytiquement la valeur de $E\left(\bar{P}_{k}\right)$, mais on peut l'encadrer en calculant une borne supérieure et une borne inférieure. La résolution du problème d'ordonnancent stochastique consiste alors à minimiser cette borne supérieure.

Une borne supérieure de l'espérance mathématique de la variance de l'erreur d'estimation d'état est donnée par la récurrence

$$
\begin{aligned}
& \Delta_{k+1}=\sum_{i=1}^{\sigma} q_{i}\left[\left(\bar{A}_{i}-\bar{K}_{i} \bar{C}_{i}\right) \Delta_{k}\left(\bar{A}_{i}-\bar{K}_{i} \bar{C}_{i}\right)^{T}+\bar{K}_{i} \bar{V}_{i} \bar{K}_{i}^{T}+\bar{W}_{i}\right] \\
& \quad \text { où } \Delta_{0}=\bar{P}_{0} .
\end{aligned}
$$

L'algorithme d'ordonnancement consiste à trouver les valeurs $\pi_{i}$ qui minimisent la borne supérieure en régime permanent, c'est-à-dire lorsque $\Delta_{k+1}=\Delta_{k}$. Afin de formuler le problème en termes d'optimisation, donc d'exprimer un critère scalaire, on peut choisir, comme précédemment, d'exprimer la minimisation de la matrice de variance en utilisant sa trace. Dans ce cas, le problème de conception peut être reformulé sous forme suivante

$$
\begin{aligned}
& \min _{\pi_{i}} \operatorname{tr}(\Delta) \\
& \text { sous les contraintes } \\
& \Delta=\sum_{i=1}^{\sigma} \pi_{i}\left[\left(\bar{A}_{i}-\bar{K}_{i} \bar{C}_{i}\right) \Delta\left(\bar{A}_{i}-\bar{K}_{i} \bar{C}_{i}\right)^{T}+\bar{K}_{i} \bar{V}_{i} \bar{K}_{i}^{T}+\bar{W}_{i}\right] \\
& \bar{K}_{i}=\bar{A}_{i}+\Delta_{i} \bar{C}_{i}^{T}\left(\bar{C}_{i} \Delta \bar{C}_{i}^{T}+\bar{V}_{i}\right)^{-1} \\
& \sum_{i=1}^{\sigma} \pi_{i}=1,0 \leqslant \pi_{i} \leqslant 1, \Delta>0
\end{aligned}
$$

Ce problème d'optimisation peut être résolu par une méthode d'optimisation (par exemple, un algorithme de gradient) ou un algorithme d'exploration de l'espace de recherche lorsqu'on est confronté à un nombre raisonnable $\sigma$ de configurations de capteurs. Après la détermination de la valeur $\pi_{i}$ des probabilités, les groupes de capteurs transmettant leur information sont activés selon les probabilités correspondantes.

\section{EXEMPLE D'ILLUSTRATION}

Considérons le système à temps discret (1) décrit par les matrices suivantes :

$$
\begin{gathered}
A=\left[\begin{array}{ccccc}
0.2 & 1 & 1 & -0.2 & 0.1 \\
0.8 & -0.5 & 1 & 0.4 & 0.2 \\
0 & 0 & 0.8 & 1 & 0.3 \\
0 & 0 & 0.8 & 0.3 & 0.4 \\
0 & 0 & 0 & 0 & 0.1
\end{array}\right], F=\left[\begin{array}{rr}
-1 & 0.2 \\
0.5 & 0 \\
0 & 0.4 \\
0 & 1 \\
1 & 0
\end{array}\right] \\
\mathcal{C}_{1}=\left[\begin{array}{ccccc}
1 & 0.1 & 0.3 & 0 & 0 \\
0 & 0.1 & 0 & -0.4 & 0 \\
0 & 0.3 & 0 & 0 & 0.1
\end{array}\right]
\end{gathered}
$$

$$
\begin{gathered}
\mathcal{C}_{2}=\left[\begin{array}{ccccc}
0 & 0 & 0.1 & 0 & 0 \\
0 & 1 & 0 & 1 & 0 \\
0.5 & 0 & 0.3 & 0 & 0.1
\end{array}\right] \\
\mathcal{C}_{3}=\left[\begin{array}{ccccc}
0 & 0 & 0 & 0 & 1 \\
0.1 & 0 & 0.1 & 1 & 0 \\
0 & 0 & 0 & 0.09 & 0.2
\end{array}\right] \\
\mathcal{C}_{4}=\left[\begin{array}{cccccc}
0 & 0 & 0.25 & 0.15 & 0 \\
0.05 & 0 & 0.05 & 0.05 & 0.01 \\
0.1 & 0 & 0 & 0.055 & 0.1
\end{array}\right], V=I \\
W=\left[\begin{array}{ccccc}
0.475 & 0 & 0 & 0 & 0 \\
0 & 0.44 & 0 & 0 & 0 \\
0 & 0 & 0.295 & 0 & 0 \\
0 & 0 & 0 & 0.435 & 0 \\
0 & 0 & 0 & 0 & 0.27
\end{array}\right]
\end{gathered}
$$

Le défaut associé à la première colonne de la matrice $F$ apparaît à l'instant $r_{1}=40$, avec $\Upsilon_{k}^{1}=10 \sin (0.5 k)$, tandis que le second défaut (associé à la deuxième colonne de $F$ ) apparaît à $r_{2}=10$ avec $\Upsilon_{k}^{2}=15$.

Nous utilisons la version sous-optimale de l'algorithme 2 (voir Remarque 2) pour calculer un ordonnancement sous-optimal des mesures. La figure 2 montre les résidus réduits de la sortie $q_{k}^{r}=\left[\begin{array}{cc}q_{k}^{r 1} & q_{k}^{r 2}\end{array}\right]^{T}$. Les deux figures de droite ont été obtenues lors de l'utilisation d'une séquence périodique arbitraire alors que les figures de gauche sont issues de l'utilisation de la séquence sous-optimale calculée avec l'algorithme proposé. Dans les deux cas, la détection et la localisation des défauts est possible, mais la solution permettant de minimiser la variance des résidus permet clairement de réduire le taux de non-détection, en particulier lors de l'apparition de défauts naissants ou de défauts de faible amplitude.
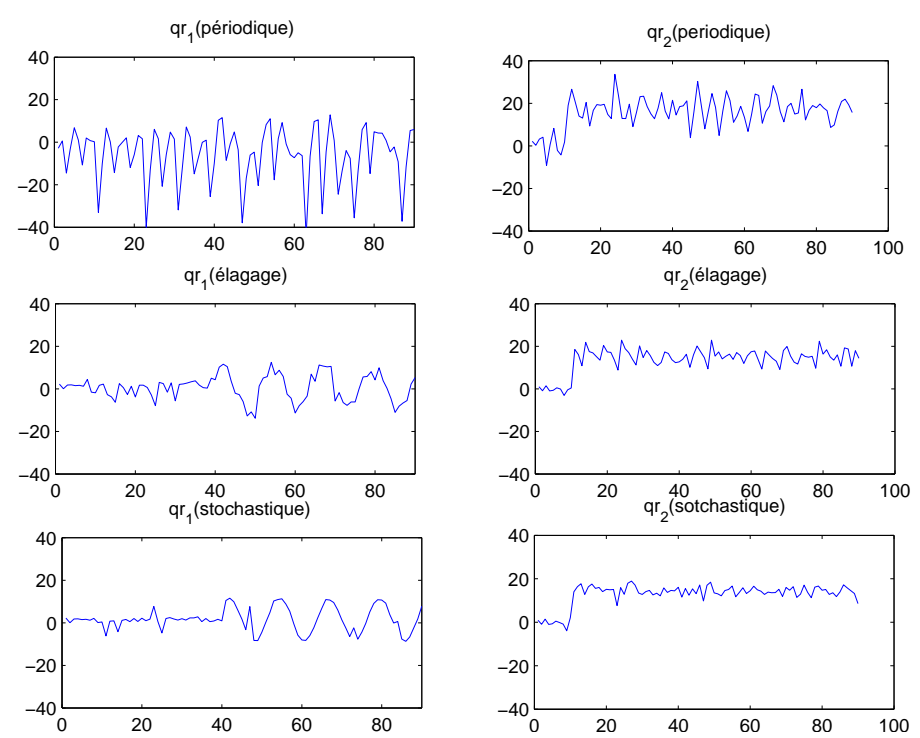

Fig. 2. Évolution des résidus

La figure 3 montre l'évolution de la trace de la matrice de covariance de l'erreur d'estimation pour les deux cas : 
un ordonnancement sous-optimal des mesures et un ordonnancement périodique arbitraire. On peut voir clairement l'avantage et la performance de la méthode proposée.

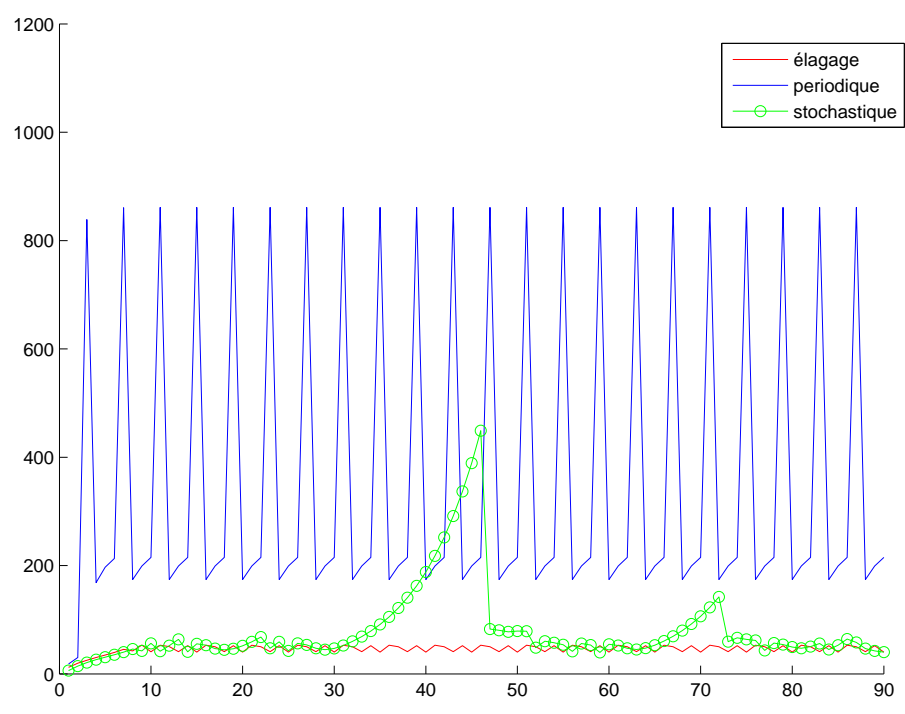

Fig. 3. Trace de la matrice de variance

\section{Conclusion}

Cet article traite le problème de détection et de localisation de défauts multiples au sein d'un système contrôlé en réseau sous contraintes de communication. Plus particulièrement, on a considéré la co-conception de l'ordonnancement des mesures et la détection-localisation des défauts avec une bande passante limitée. La séquence de commutation est calculée de façon à assurer la détectabilité et l'isolabilité des défauts tout en minimisant l'effet du bruit de mesure. Nous proposons dans le futur d'étendre cette étude pour un horizon de temps infini en utilisant des techniques d'ordonnancement en ligne.

\section{RÉFÉRENCES}

[1] Chabir K., Sauter D., Ben Gayed M., Abdelkrim M.N. Design of an adaptive Kalman filter for fault detection of Networked Control Systems. 16th Mediterranean Conference on Control and Automation, Ajaccio, France, June 25-27, 2008.

[2] Gomez-Esterm F., Canudas-de-Wit C., Rubio F.R Adaptive delta modulation in networked controlled systems with bounded disturbances. IEEE Transactions on Automatic Control, vol. 56, n 1, pp. 129-134, January 2011.

[3] Gupta V., Chung T.H., Hassibi B., Murray R.M. On a stochastic sensor selection algorithm with applications in sensor scheduling and sensor coverage. Automatica, vol. 42, n 2 , pp. 252-260, February 2006.

[4] Hashemi Nejad H., Sauter D., Aberkane S., Aubrun C. Fault detection and isolation in networked control systems with access constraints and packet dropouts. 19th Mediterranean Conference on Control and Automation, MED 2011, Corfu, Greece, 20-23 June 2001

[5] Heemels W.P.M.H., Teel A.R., Van de Wouw N., Nesic D., Networked control systems with communication constraints : tradeoffs between transmission intervals, delays and performance. IEEE Transactions on Automatic Control, vol. 55, $\mathrm{n}^{\circ}$ 8, pp. 1781-1796, August 2010.

[6] Hristu-Varsakelis D. Short-period communication and the role of zero-order hHolding in networked control systems. IEEE Transactions on Automatic Control, vol. 53, n ${ }^{\circ}$ 5, pp. 1285-1290, June 2008.

[7] Keller J.Y. Fault isolation filter design for linear stochastic systems. Automatica, vol. 35, n 10, October 1999.
[8] Li J.,Tang G.Y. Fault diagnosis for networked control systems with delayed measurements and inputs. IET Control Theory Applications, vol. 4, n 6, pp. 1047-1054, June 2010.

[9] Li S., Xu B. Modified fault isolation filter for networked control system with send-on-delta sampling. 30th Chinese Control Conference, Yantai, China, July 22-24, 2011.

[10] Li Z., Wang W., Jiang Y. Intelligent scheduling and optimisation for resource-constrained networks. IET Control Theory and Applications, vol. 4, n 12, pp. 2982-2992, December 2010.

[11] Li W., Zhu Z., Ding S.X. Fault detection design of networked control systems. IET Control Theory and Applications, vol. 5, $\mathrm{n}^{\circ}$ 12, pp. 1439-1449, August 2011.

[12] Mao Z., Jiang B., Shi P. $H_{\infty}$ fault detection filter design for networked control systems modeled by discrete Markovian jump systems. IET Control Theory and Applications, vol. 1, n 5, pp. 1336-1343, September 2007.

[13] Mao Z., Jiang B., Shi P. Protocol and fault detection design for nonlinear networked control systems. IEEE Transactions on Circuits and Systems, II Express Briefs, vol. 56, n 3, pp. 255259, March 2009.

[14] Ping Z., Ding S.X. Influence of sampling period on a class of optimal fault-detection performance. IEEE Transactions on Automatic Control, vol. 54, n 6, pp. 1396-1402, June 2009.

[15] Shi L., Peng C., Chen J. Sensor data scheduling for optimal state estimation with communication energy constraint. Automatica, vol. 47, $\mathrm{n}^{\circ}$ 8, August 2011.

[16] Song H., Yu L., Zhang W.A. Stabilisation of networked control systems with communication constraints and multiple distributed transmission delays. IET Control Theory and Applications, vol. 3, n 10, pp. 1307-1316, October 2009.

[17] Vitus M.P., Zhang W., Abate A., Hu J., Tomlin C.J. On efficient sensor scheduling for linear dynamical systems. American Control Conference, ACC 2010, Baltimore, Maryland, USA, June 30 - July 2, 2010.

[18] Wang Y., Ding S.X., Ye H., Wang G. A new fault detection scheme for networked control systems subject to uncertain timevarying delay. IEEE Transactions on Signal Processing, vol. 56, n 10 , pp. 5258-5268, October 2008.

[19] Wang Y.Q., Ye H., Ding S.X., Wang G.Z., Zhou D.H. Residual generation and evaluation of networked control systems subject to random packet dropout. Automatica, vol. 45 , n 10 , pp. $2427-$ 2434, 2009.

[20] Zhang W., Branicky M.S., Phillips S.M. Stability of networked control systems. IEEE Control Systems, vol. 21, n ${ }^{\circ}$ 1, pp. 84-99, February 2001.

[21] Zhang P., Ding S.X., Frank P.M., Sader M. Fault detection of networked control systems with missing measurements. 5th Asian Control Conference, Melbourne, Australia, July 20-23, 2004.

[22] Zhang W., Jianghai H., Lian J. Quadratic optimal control of switched linear stochastic systems. Systems \&3 Control Letters, vol. 59, n 11, pp. 736-744, November 2010. 